\title{
Comparison of the Optical Planar Waveguide Sensors' Characteristics Based on Guided-Mode Resonance
}

\author{
S. Bellucci ${ }^{1, *(\mathbb{D}}$, V. Fitio ${ }^{2}$, I. Yaremchuk ${ }^{2} \mathbb{D}$, O. Vernyhor $^{2}$, A. Bendziak $^{2}$ and Y. Bobitski ${ }^{2,3}$ \\ 1 INFN-Laboratori Nazionali di Frascati, Via E. Fermi 40, 00044 Frascati, Italy \\ 2 Department of Photonics, Lviv Polytechnic National University, S. Bandera Str.12, 79013 Lviv, Ukraine; \\ volodymyr.m.fito@lpnu.ua (V.F.); yaremchuk@polynet.lviv.ua (I.Y.); vernyhor@gmail.com (O.V.); \\ andrii.v.bendziak@lpnu.ua (A.B.); bobitski@polynet.lviv.ua (Y.B.) \\ 3 Department of Physics, University of Rzeszow, Pigonia Str.1, 35959 Rzeszow, Poland \\ * Correspondence: stefano.bellucci@lnf.infn.it
}

Received: 1 July 2020; Accepted: 28 July 2020; Published: 6 August 2020

\begin{abstract}
A comparison of optical sensors' characteristics based on guided-mode resonance has been carried out. It was considered a prism structure with a metal film, a metal grating on a metal substrate and a dielectric grating on a dielectric substrate. It is shown that the main characteristics are determined by the sensitivity of the constant propagation of the respective waveguides on a change in wavelength and a change in the refractive index of the tested medium. In addition, they depend on the full width at half maximum of the spectral or angular reflectance dependence. The corresponding analytical relationships obtained for the three types of sensors are almost the same. It is demonstrated that the ratio of the sensor spectral sensitivity on the resonance curve spectral width is equal to the ratio of the angular sensitivity on the angular width of the corresponding resonance curve for all three types of sensors.
\end{abstract}

Keywords: sensor; prism structure; grating; waveguide; resonance; sensitivity

\section{Introduction}

Optical sensors for measuring biological solutions' refractive indices are mainly based on resonant excitation of the waveguide processes. The extensive reviews [1,2] describe various structures, which are suitable for dynamically measuring the liquids' and gases' refractive indices. Sensors based on the resonant excitation of the waveguide processes can be divided into two types. There is resonant excitation of surface plasmon-polariton waves at the metal-dielectric interface [3] and resonant excitation of waveguide modes in dielectric gratings [4]. Resonant excitation of surface plasmon-polariton waves at the metal-dielectric interface is possible using prism structures [5-7]. In this case, a thin metal film (gold or silver) deposited on the prism contacts with the tested medium. Moreover, the surface plasmon-polariton resonance can be excited using metal or dielectric grating on the metal substrate [7-9]. In fact, the above described sensor structures [3-9] are based on planar waveguides, which are easy to manufacture with modern lithography technology.

In addition to sensors based on planar waveguides, a number of sensors based on optical fibers have been proposed [1,10-13]. The sensors, in particular, with an optical fiber Bragg grating (FBG) are considered in [10]. Sensors based on an optical fiber in which the side is polished are studied in [11]. Fiber sensors based on Long-Period Fiber Grating are discussed in detail in [12], and sensors based on D-type fiber are described in [13]. In general, a large number of sensors based on optical fibers have been proposed, the sensitive elements of which are characterized by small dimensions. The attained refractive index detection limit of $2.5 \times 10^{-8}$ is so far the highest value reported for any SPR-based sensor [14]. The variety of fiber sensors is much higher than that based on planar waveguides, but their 
fabrication is much more complicated, as evidenced by the overwhelming number of theoretical works using numerical methods.

The reflection coefficient of the grating can be equal to unity at a careful selection of the measured structure parameters under resonant excitation of the waveguide mode in dielectric gratings $[4,15]$. The resonance is disturbed when the refractive index of the tested medium changes. As a result, the reflection coefficient decreases. However, it can be restored at the different wavelength or at the different light angle of incidence on the grating [16,17].

In the case of surface plasmon-polariton resonance, the reflection coefficient is zero at the carefully selected prism or grating structure parameters $[3,8,9]$. The reflection coefficient becomes nonzero when the resonance is disturbed due to the change in the tested liquid refractive index. It is restored at the different wavelength at the constant angle of incidence, or at the different angle of incidence at the constant wavelength.

The value of the shifts of resonance minima or maxima determines the sensors sensitivity on the change in the tested media refractive index. Therefore, a number of studies are devoted to increasing sensitivity. In particular, prism structures were analyzed in [18-24]. In [18], the application of a layer of perforated dielectric on the metal film in the prism structure was proposed, which slightly increased sensitivity. In this case, the structure can operate as a sensor not only for TM (transverse magnetic) polarized waves, but also for TE (transverse electric) ones. In [19], prism structures with and without the additional dielectric layer deposited on the metal film were studied. The structure with the additional layer can operate for both the TM and the TE polarized waves. It was found that the sensitivity is decreased when the additional dielectric layer is used. It decreases significantly for TM polarized waves. The sensitivity of TE polarized waves appears, although it is less than for TM polarized waves without the additional dielectric layer. It was also indicated that sensitivity increases when prism refractive index decreases.

It was proposed to arrange a thin dielectric layer with the refractive index lower than the prism refractive index in the prism structure between the prism and the metal film [21]. The spectral and angular dependences of the reflection coefficient have narrow and wide resonances in such a structure. The narrow one is observed at a shorter wavelength. However, such an innovation did not increase the sensor sensitivity. It is only possible to increase the reliability of fixing the values of the angles or wavelengths at which the reflection coefficient minima are observed. In [22], it was proposed to deposit the gold grating with a period several times smaller than the wavelength on the gold film. This resulted in a spectral narrowing of the resonance curve by 3-4 times. The main characteristics of sensors based on the Otto and Kretschmann configurations, namely detection speed, sensitivity, portability, are given in [23].

The results of the study of surface plasmon-polariton resonance sensors based on grating structures are presented in a huge number of the scientific works. Here are given only a few of them, the most original ones in our opinion [24-31]. The multiple surface plasmon spectroscopy for the study of biomolecular systems was considered in [24]. A biosensor on a relief grating with a leaky mode was studied in [25]. For gas studies, it was proposed to use the grating-based structure in which surface plasmon-polariton resonance exists [26,27]. An original grating formation technology was developed in [28]. Usage of digital versatile discs for studying Raman spectroscopy was described in [29]. J. Homola and coauthors compared the sensitivity of the surface plasmon-polariton resonance sensors based on prism and grating structures [30]. It was shown that the spectral sensitivity is higher for prism structures, and the angular sensitivities are approximately the same. The compact and low-cost biosensor based on novel approach to spectroscopy of surface plasmons was considered in [31].

Guided-mode resonance in the dielectric gratings on dielectric substrates has been described in many works, in particular $[4,15,16,18,32,33]$. The studies of bulk gratings at normal incidence of the laser beam are presented in [4]. The average grating refractive index is higher than refractive indices of the substrate and the surrounding medium. Therefore, such a structure is an asymmetric planar waveguide [34]. As a result, the guided-mode resonance occurs at carefully selected periodic structure 
parameters and the reflection coefficient of grating becomes equal to 100 percent. The spectral width of the resonance band decreases when modulation of the grating refractive index decreases [4]. It was also found that the spectral bandwidth of the resonance for TM polarized waves is much narrower than the resonance width for TE polarized waves. Mostly, such gratings can be formed on photopolymer compositions by the holographic method, and the maximum amplitude of refractive index modulation upon exposure is no more than 0.017 [35]. The theories of the light beam interaction with structures in which a homogeneous dielectric layer is deposited on the dielectric substrate, on which a dielectric relief grating is formed, the thickness of which is much smaller than the thickness of the dielectric layer, and the grating period is significantly less than the length waves of test radiation, were developed and considered in $[16,17]$. The developed theories are quite complex, however, they allow the calculation of the reflection coefficient of the periodic structure. The experimental studies of waveguide structures with dielectric gratings are presented in [32]. It is shown that the spectral dependence consists of resonance peaks of high reflection, which are significantly larger than the Fresnel reflection. However, the reflection coefficient is much less than unity. It was also experimentally shown that the maximum transmission peaks on the spectral characteristic shift when the refractive index of the aqueous solution changes. The results of theoretical and experimental studies of the waveguide structure with the dielectric layer on which the sinusoidal dielectric grating was formed, are described in [33]. Theoretical predictions were confirmed by the numerical calculations. The experimentally measured reflection coefficients under resonance are less than unity. The spectral width of the resonance is much wider than in numerical calculation. However, wavelengths of the maximum reflection are matched for both cases. Such a discrepancy between theoretical and experimental results can be explained by the fact that strict periodicity is disturbed in the manufacture of relief gratings by etching.

The application of the aforementioned planar optical sensors in biological and chemical sensing has obtained extensive focus. Detection of small amounts of toxic species and contaminants is critical for environmental monitoring, human health improvement as well as the prevention of biological and chemical warfare threats. Therefore, the study and improvement of optical sensors has a high priority.

The operation of all three types of planar waveguide sensors is based on the resonant excitation of waveguide processes. The propagation constant in the waveguide is determined by the wavelength and the refractive indices of the layers form the waveguide; in particular, the refractive index of the tested medium. Therefore, the sensitivity of the propagation constant changes upon changing the wavelength and, on the change in the tested medium refractive index, it can give information about the sensitivity of the sensors. In the present work, the analytical equations relating the parameters of the waveguide, the angle of incidence, the angular and spectral sensitivity of the sensor, and the relationship between the full widths at half maximum of the resonance spectral and angular dependences, will be obtained. These studies will show us what to expect from sensors based on planar waveguides.

\section{Spectral and Angular Sensors' Sensitivities and Their Relationship with Planar Waveguide Parameters}

The simple structures of the sensitive elements of sensors based on resonance phenomena in planar waveguides are shown in Figure 1.

In the case that the prism structure is under resonance, the following equation must be satisfied as follows [22]:

$$
\frac{2 \pi n}{\lambda} \sin \theta=\beta\left(\lambda, n_{a}\right),
$$

where $\lambda$ is wavelength, $\beta$ is propagation constant.

The equality in Equation (1) is strong under resonance [22,36]. Therefore, the propagation constant $\beta$ is a real value. Equation (1) allows the determination of the resonant angle of incidence $\theta$ of a plane wave on the metal film. This equation is accurate since the interfaces between the metal film and the dielectric layers are plane. 
For the grating structure, which is shown in Figure $1 \mathrm{~b}$ under surface plasmon-polariton resonance, the following approximate equation is true [3]:

$$
\frac{2 \pi n}{\lambda} \sin \theta \pm \frac{2 \pi}{\Lambda} \cong \pm \frac{2 \pi n_{a}}{\lambda} \operatorname{Re}\left(\sqrt{\frac{\varepsilon_{m}}{\varepsilon_{m}+n_{a}^{2}}}\right)
$$

The smaller the perturbations of the plane interface between the metal and the dielectric, that is, the smaller the grating thickness $d$, the smaller the difference between the right and left sides of Equation (2) under resonance. The relations $\left|\operatorname{Re}\left(\varepsilon_{m}\right)\right| \gg \operatorname{Im}\left(\varepsilon_{m}\right)$ and $\left|\operatorname{Re}\left(\varepsilon_{m}\right)\right| \gg \varepsilon_{a}[37,38]$ are true for silver or gold, taking into account that resonance is most pronounced in periodic structures with these metals. In this case, the following relation also is satisfied $F(\lambda)=\sqrt{\frac{\varepsilon_{m}(\lambda)}{\varepsilon_{m}(\lambda)+\varepsilon_{d}}} \cong 1$. There is at $\lambda>1 \mu \mathrm{m}$, $1<\operatorname{Re}(F(\lambda))<1.03$ for silver and $\operatorname{Im}(F(\lambda))<0.001$ for water refractive index $n_{a}=1.3242[39,40]$. Therefore, the Equation (2) for $n=1$ can be rewritten as follows:

$$
\frac{2 \pi}{\lambda} \sin \theta \pm \frac{2 \pi}{\Lambda} \cong \pm \frac{2 \pi n_{a}}{\lambda} .
$$

When the guided-mode resonance is excited in the grating structure, the following equation is satisfied [4]:

$$
\frac{2 \pi}{\lambda} \sin \theta \pm \frac{2 \pi}{\Lambda} \cong \pm \beta\left(\lambda, n_{\mathrm{a}}\right) .
$$

The propagation constant is approximately $15 \mu^{-1}$ for our waveguide parameters, which consist of the grating, the substrate and the tested medium. The difference between the right and left sides of Equations (1) and (3) is less than $0.001 \mu \mathrm{m}^{-1}$. The less the modulation of the grating refractive index, the smaller the difference between the right and left sides of Equation (4) [4]. Discrete propagation constants in the planar dielectric waveguides can be determined by the method described in [34], as well as by the numerical method in the frequency domain, which provides precision calculation accuracy [41,42]. All calculations for three structures shown in Figure 1 were carried out for the resonance wavelength of $1.064 \mu \mathrm{m}$.
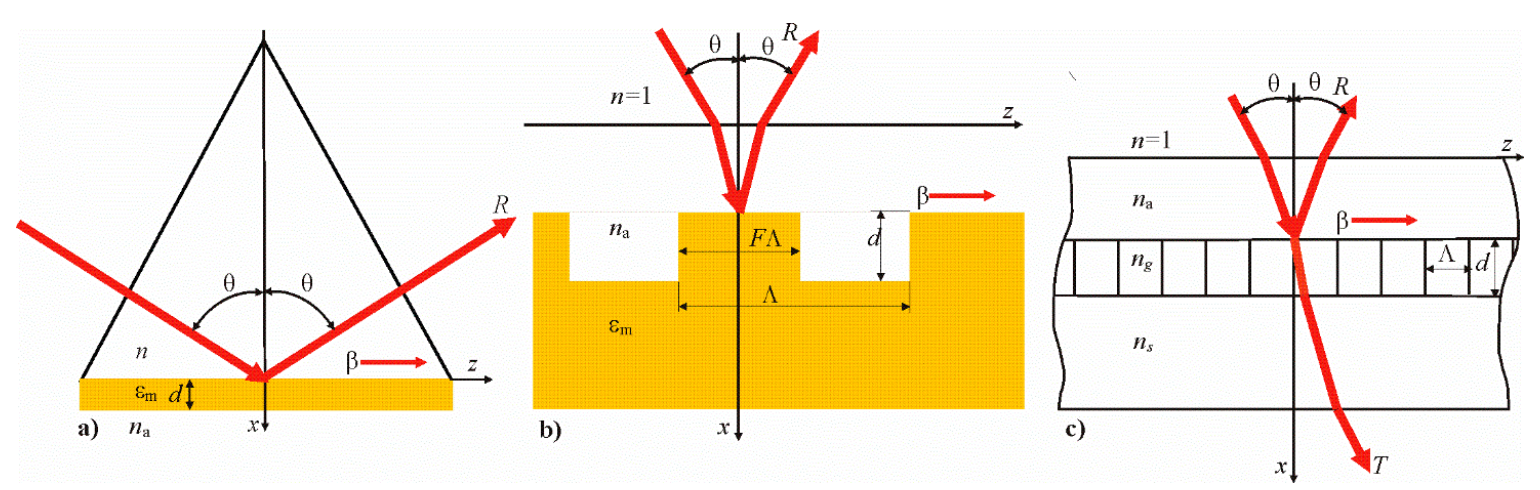

Figure 1. Sensitive elements of the sensors, where $\varepsilon_{m}$ is the dielectric constant of the metal, $n_{a}$ is the test medium refractive index, $d$ is the metal film thickness or the grating thickness, $\Lambda$ is the grating period, $\beta$ is the propagation constant of the waveguide modes under resonance. (a) is the prism structure, where $n$ is the prism refractive index, $\theta$ is the angle of incidence of the light beam. (b) is the metal grating on the metal substrate, where $F$ is the fill factor, which, in our studies, is equal to 0.5 . (c) is the dielectric grating-based structure, where $n_{g}$ is the average refractive index of the dielectric grating, $n_{S}$ is the refractive index of the substrate. $\theta$ is the angle of incidence of the light beam in the air with the refractive index $n=1$ for Figure 1a,b. 
2.1. Relationship Between the Parameters of the Sensor Based on the Prism Structure and the Properties of the Planar Waveguide Dielectric/Thin Metal Film/Dielectric

Let consider the case of surface plasmon-polariton resonance in the prism structure. The prism structure is realized with the following parameters, namely $n=1.56, n_{a}=1.3242, \lambda=1.064 \mu \mathrm{m}$, $\theta \approx 1.040217 \mathrm{rad}, d \approx 52.6967 \mathrm{~nm}$. The reflection coefficient is $<1.5 \times 10^{-9}$ for such parameters. It was calculated by the matrix method [43]. The resonance angle of incidence on the metal film and the metal film thickness in the prism structure were determined from the condition that the real and imaginary parts of the reflection coefficient $\operatorname{Re}(r(\mathrm{~d}, \theta))=0, \operatorname{Im}(r(d, \theta))=0$. The intersection of these curves determines the resonant thickness of the metal film and the light beam angle of incidence on the film as shown in Figure 2.

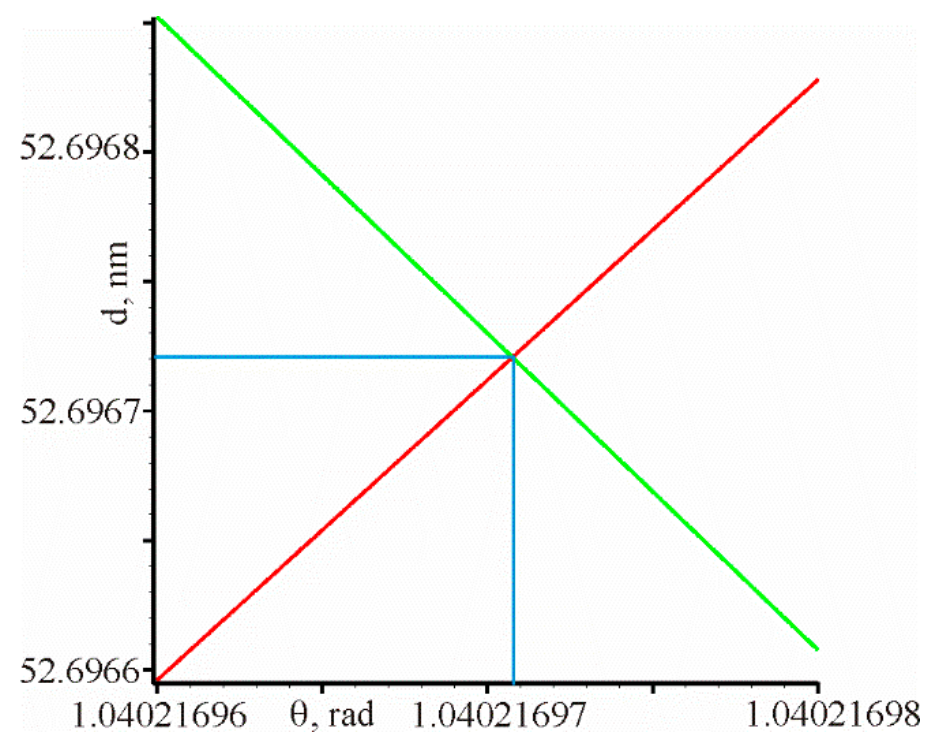

Figure 2. Determination of the resonant thickness of the metal film and the angle of incidence on the film with the following parameters $=1.56, n_{a}=1.3242, \lambda=1.064 \mu \mathrm{m}$.

Propagation constant $\beta$ can be determined on the basis of the following equation [44]:

$$
B(x, y)=\exp \left(-2 k_{m} d\right)-\frac{k_{m} / \varepsilon_{m}+k / \varepsilon}{k_{m} / \varepsilon_{m}-k / \varepsilon} \times \frac{k_{m} / \varepsilon_{m}+k_{a} / \varepsilon_{a}}{k_{m} / \varepsilon_{m}-k_{a} / \varepsilon_{a}}=0
$$

where $x$ and $y$ are real and imaginary parts of the propagation constant, respectively. That is $\beta=x+i y$, $k_{m}^{2}=\beta^{2}-k_{0}^{2} \varepsilon_{m}, k^{2}=\beta^{2}-k_{0}^{2} \varepsilon, k_{a}^{2}=\beta^{2}-k_{0}^{2} \varepsilon_{a}, k_{0}^{2}=\left(\frac{2 \pi}{\lambda}\right)^{2}$.

The function $B(x, y)$ is complex in the general case. It will be equal to zero if the real and imaginary parts of this function are also equal to zero. Therefore, the intersection of the curves must satisfy the following equations:

$$
\operatorname{Re}(B(x, y))=0, \operatorname{Im}(B(x, y))=0,
$$

which determine searched propagation constants. The corresponding graphical dependencies are shown in Figure 3. The two propagation constants were obtained using systems of Equation (6). One propagation constant is complex. The second one is real and equals $\beta=7.945642 \mu \mathrm{m}^{-1}$. The angle $\theta$ calculated by Equation (1) is $\theta=1.040217 \mathrm{rad}$ for $\beta=7.945642 \mu \mathrm{m}^{-1}$. The reflection coefficient $\mathrm{R}$ of the metal film is less than $1.5 \times 10^{-9}$ at this angle. Therefore, it can be argued that Equation (1) is accurate under plasmon-polariton resonance in the prism structure. 


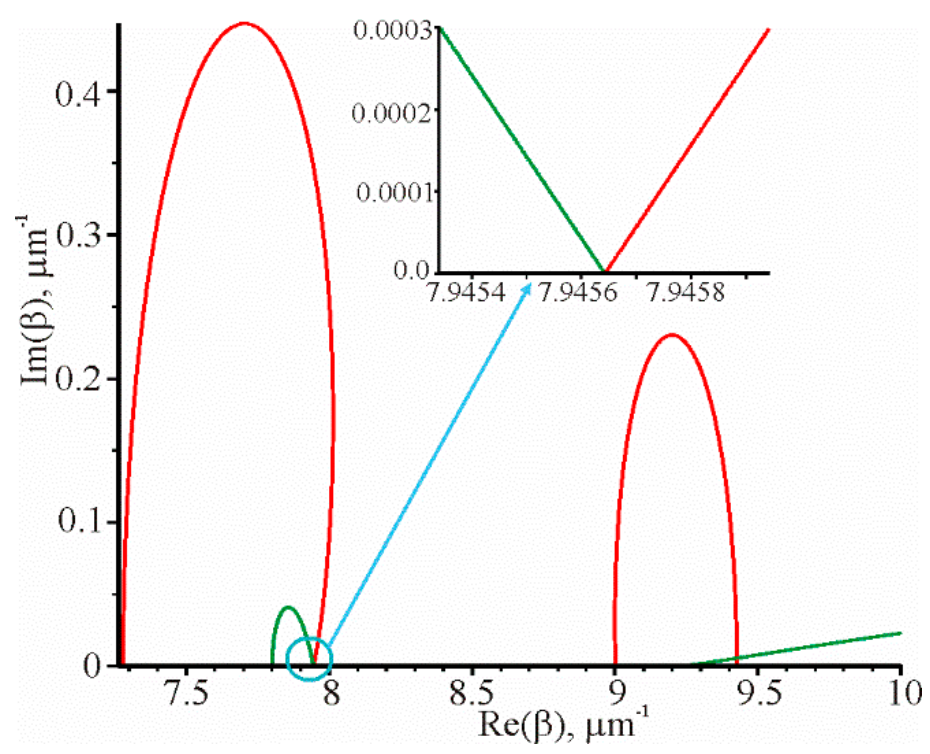

Figure 3. Graphical dependences $\operatorname{Re}(B(x, y))=0$ (red curve) and $\operatorname{Im}(B(x, y))=0$ (green curve) for the following parameters, $\lambda=1.064 \mu m, \theta=1.040217 \mathrm{rad}, d=52.697 \mathrm{~nm}, n=1.56, n_{a}=1.3242$. The inset shows the progress of the green and red curves on an enlarged scale.

The left side of Equation (1) during of the measurement process can depend on the angle $\theta$ and wavelength $\lambda$. The right side depends on $\lambda$ and the tested medium refractive index $n_{a}$. It is possible to establish a connection between the waveguide parameters using Equation (1), which personifies the right side of the equation, with the sensor characteristics as a whole.

Let the angle of incidence be fixed, in this case when $n_{a}$ changes to $d n_{a}$, the resonance wavelength changes on $\mathrm{d} \lambda$. Accordingly, the change in the right and left sides of Equation (1) can be written as follows:

$$
-\frac{2 \pi n}{\lambda^{2}} \sin \theta d \lambda=\frac{\partial \beta}{\partial n_{a}} d n_{a}+\frac{\partial \beta}{\partial \lambda} d \lambda
$$

Let's introduce the following notation $S_{n}^{(\beta)}=\frac{\partial \beta}{\partial n_{a}}, S_{\lambda}^{(\beta)}=\frac{\partial \beta}{\partial \lambda}$. The $S_{\lambda}^{(\beta)}$ and $S_{n}^{(\beta)}$ can be numerically determined using Equation (5) as follows:

$$
\begin{gathered}
S_{\lambda}^{(\beta)}=\frac{\beta\left(\lambda_{r e z}+\Delta \lambda, n_{a}\right)-\beta\left(\lambda_{r e z}-\Delta \lambda, n_{a}\right)}{2 \Delta \lambda}, \\
S_{n}^{(\beta)}=\frac{\beta\left(\lambda_{r e z}, n_{a}+\Delta n_{a}\right)-\beta\left(\lambda_{r e z}, n_{a}-\Delta n_{a}\right)}{2 \Delta n_{a}},
\end{gathered}
$$

From Equation (7), after simple algebraic transformations taking into account Equation (1), the spectral sensitivity can be found as the ratio of the change in the resonance wavelength on the change in the tested medium refractive index at the constant $\theta$ as follows:

$$
S_{\lambda}=\frac{d \lambda}{d n_{a}}=-\frac{S_{n}^{(\beta)} \lambda}{S_{\lambda}^{(\beta)} \lambda+\beta} .
$$

The resonance angle $\theta$ changes when the tested medium refractive index changes at the fixed wavelength. Based on Equation (1), the following equation can be obtained for this case:

$$
\frac{2 \pi n}{\lambda} \cos \theta d \theta=\frac{\partial \beta}{\partial n_{a}} d n_{a}
$$


The angular sensitivity can be found from Equation (11) as the ratio of the change in the resonance angle on the change in the tested medium refractive index:

$$
S_{\theta}=\frac{d \theta}{d n_{a}}=\frac{S_{n}^{(\beta)} \lambda}{2 \pi n \cos \theta} .
$$

The change in the resonant wavelength by $\delta \lambda$ on the basis of Equation (1) at the constant refractive index $n_{a}$, and with the change in the corresponding resonant angle by $\delta \theta$, can be determined using the next equation:

$$
-\frac{2 \pi n}{\lambda^{2}} \sin \theta \delta \lambda+\frac{2 \pi n}{\lambda} \cos \theta \delta \theta=\frac{\partial \beta}{\partial \lambda} \delta \lambda
$$

In fact, based on Equation (13), the relation can be calculated between the full widths at half maximum of the spectral resonance curve $\delta \lambda_{0.5}$ and the angular resonance curve $\delta \theta_{0.5}$. This ratio can be written as follows:

$$
\delta \lambda_{0.5}=-\frac{2 \pi n \cos \theta}{S_{\lambda}^{(\beta)} \lambda+\beta} \delta \theta_{0.5}
$$

Let the right side of the Equation (10) be divided by the right side of the Equation (14); as a result, the following can be obtained:

$$
\frac{S_{\lambda}}{\delta \lambda_{0.5}}=\frac{S_{\theta}}{\delta \theta_{0.5}}=\frac{\lambda S_{n}^{(\beta)}}{2 \pi n \cos \theta \delta \theta_{0.5}} .
$$

\subsection{Sensitivity of the Sensor Based on the Metal Grating under Resonant Excitation of the Surface} Plasmon-Polariton Wave

The sensitivities $S_{\lambda}, S_{\theta}$, as well as the relationship between $\delta \lambda_{0.5}$ and $\delta \theta_{0.5}$ under surface plasmon-polariton resonance in the grating structure (Figure 1b), by doing the same using Equation (2), can be determined as follows:

$$
\begin{gathered}
S_{\lambda+}=S_{\lambda-}=\frac{d \lambda}{d n_{a}}=\frac{\lambda}{\sin \theta \mp n_{a}}=\Lambda, \\
S_{\theta \pm .}=\frac{d \theta}{d n_{a}}= \pm \frac{1}{\cos \theta}, \\
\delta \lambda_{0.5}=\Lambda \cos \theta \delta \theta_{0.5} .
\end{gathered}
$$
written as:

Comparing the ratios $\frac{S_{\lambda}}{\delta \lambda_{0.5}}=\frac{\Lambda}{\Lambda \cos \theta \delta \theta_{0.5}}$ and $\frac{S_{\theta}}{\delta \theta_{0.5}}=\frac{\frac{1}{\cos \theta}}{\delta \theta_{0.5}}$, it can be seen that these ratios can be

$$
\frac{S_{\lambda}}{\delta \lambda_{0.5}}=\frac{S_{\theta}}{\delta \theta_{0.5}}=\frac{1}{\cos \theta \delta \theta_{0.5}} .
$$

\subsection{Sensitivity of the Sensor Based on the Dielectric Grating under Resonant Excitation of the Localized} Waveguide Mode

The sensitivities $S_{\lambda}, S_{\theta}$, and the relationship between $\delta \lambda_{0.5}$ and $\delta \theta_{0.5}$ under guided-mode resonance in the grating structure (Figure 1c), by doing the same using Equation (3), can be determined as follows:

$$
\begin{gathered}
S_{\lambda+}=\frac{\delta \lambda}{\delta n_{\mathrm{a}}}=-\frac{S_{n}^{(\beta)}}{\frac{2 \pi \sin \theta}{\lambda^{2}}+S_{\lambda}^{(\beta)}} \\
S_{\lambda-}=\frac{\delta \lambda}{\delta n_{a}}=\frac{S_{n}^{(\beta)}}{\frac{2 \pi \sin \theta}{\lambda^{2}}-S_{\lambda}^{(\beta)}},
\end{gathered}
$$


where $S_{\lambda}^{(\beta)}$ and $S_{n}^{(\beta)}$ can be numerically determined from Equations (7) and (8).

The propagation constant can also be expressed as follows: $\beta\left(n_{a}, \lambda\right)=\frac{2 \pi n_{e f}}{\lambda}$. The relation $\max \left(n_{a}, n_{s}\right)<n_{e f}<n_{g}$ is fulfilled. If it is taken into account that $n_{e f}$ depends a little on the wavelength, then it can be written:

$$
S_{\lambda}^{(\beta)}=\frac{d \beta}{d \lambda} \cong-\frac{2 \pi n_{e f}}{\lambda^{2}}=-\frac{\beta}{\lambda} .
$$

After substituting (22) into (20) and (21), it can be obtained:

$$
\begin{gathered}
S_{\lambda+}=\frac{\delta \lambda}{\delta n_{\mathrm{a}}}=-\frac{S_{n}^{(\beta)}}{\frac{2 \pi \sin \theta}{\lambda^{2}}-\frac{\beta}{\lambda}}, \\
S_{\lambda-}=\frac{\delta \lambda}{\delta n_{a}}=\frac{S_{n}^{(\beta)}}{\frac{2 \pi \sin \theta}{\lambda^{2}}+\frac{\beta}{\lambda}} .
\end{gathered}
$$

Taking into account Equations (4), (22) and (23) it can be rewritten as follows:

$$
S_{\lambda \pm}=\frac{\delta \lambda}{\delta n_{\mathrm{a}}}=\mp \frac{\lambda \Lambda}{2 \pi} S_{n}^{(\beta)} .
$$

The sensitivity $S$ will be negative when there is a " + " sign in Equation (4). It is due to the fact that $S_{\lambda}^{(\beta)}$ is less than zero and the resonant wavelength will be shorter than the resonant wavelength at the normal incidence [4]. The sensitivity $S$ will be positive when there is " - " sign in Equation (4). The resonant wavelength will be longer than the resonant wavelength at the normal incidence. It should be noted that the sensitivity using the "-" sign in Equation (4) will be higher than the modulo sensitivity using the " + " sign. It is matched with the results presented in [33].

Other sensor characteristics can be written as follows:

$$
\begin{gathered}
S_{\theta \pm}=\frac{\lambda S_{n}^{(\beta)}}{2 \pi \cos \theta} . \\
\delta \lambda_{0.5}=\Lambda \cos \theta \delta \theta_{0.5} .
\end{gathered}
$$

Equation (27) is written in [45] as follows:

$$
\frac{S_{\lambda}}{\delta \lambda_{0.5}}=\frac{S_{\theta}}{\delta \theta_{0.5}}=\frac{\lambda S_{n}^{(\beta)}}{2 \pi \cos \theta \delta \theta_{0.5}}
$$

\subsection{Comparison of the Properties of Sensors based on Planar Waveguides}

It can be seen that for all types of structures in which resonant excitation occurs, the next relation is satisfied:

$$
\frac{S_{\lambda}}{\delta \lambda_{0.5}}=\frac{S_{\theta}}{\delta \theta_{0.5}}
$$

This equation is important for sensors because it determines the sensor suitability for measuring changes in the tested medium refractive index. The larger this value, the more accurate and reliable it is possible to measure the change in the refractive index.

The equations relating the characteristics of the sensitive elements of the three types of sensors with the parameters of the grating and the corresponding waveguide are presented in Table 1.

Some of the equations in the columns are the same, as can be seen from Table 1. For instance, the equations for Figure 1a,c are the same in columns 2 and 4. There is also a match for the third column for Figure 1b,c. However, the equations of the fifth row become the equations of the fourth row if it is taken into account that $S_{n}^{(\beta)}=2 \pi / \lambda[46]$ for the grating shown in Figure 1b. On the other hand, 
the conditional period $\Lambda_{\text {con }}$ can be introduced for the equations, which relates to the prism structure. This period can be determined as follows:

$$
-\frac{2 \pi}{\Lambda_{\text {con }}}=\lambda S_{\lambda}^{(\beta)}+\beta
$$

Table 1. Analytical expressions connecting the characteristics of the sensitive elements of the three types of sensors with the parameters of the grating and the corresponding waveguide.

\begin{tabular}{ccccc}
\hline Parameter & $S_{\lambda}$ & $S_{\boldsymbol{\theta}}$ & $\delta \lambda_{0.5}$ & $\frac{S_{\lambda}}{\delta \lambda_{0.5}}=\frac{S_{\theta}}{\delta \theta_{0.5}}$ \\
\hline No & $\mathbf{1}$ & $\mathbf{2}$ & $\mathbf{3}$ & $\mathbf{4}$ \\
\hline Figure 1a & $-\frac{\lambda S_{\beta}^{(\beta)}}{\lambda S_{\lambda}^{(\beta)}+\beta}$ & $\frac{\lambda S_{n}^{(\beta)}}{2 \pi n \cos \theta}$ & $\frac{2 \pi n \cos \theta}{\lambda S_{\lambda}^{(\beta)}+\beta} \delta \theta_{0.5}$ & $\frac{\lambda S_{n}^{(\beta)}}{2 \pi n \cos \theta \delta \theta_{0.5}}$ \\
\hline Figure 1b & $\Lambda$ & $\frac{1}{\cos \theta}$ & $\Lambda \cos \theta \delta \theta_{0.5}$ & $\frac{1}{\cos \theta \delta \theta_{0.5}}$ \\
\hline Figure 1c & $\frac{\lambda \Lambda}{2 \pi} S_{n}^{(\beta)}$ & $\frac{\lambda S_{n}^{(\beta)}}{2 \pi \cos \theta}$ & $\Lambda \cos \theta \delta \theta_{0.5}$ & $\frac{\lambda S_{n}^{(\beta)}}{2 \pi \cos \theta \delta \theta_{0.5}}$ \\
\hline
\end{tabular}

Thus, the equations in the columns take the same form, which coincides with the equations of the fifth row of Table 1 and corresponds to the periodic structure shown in Figure 1c.

As follows from Table 1, the sensors' characteristics are determined by the sensitivity of the propagation constants of the waveguides $\beta$ on the change in the wavelength $S_{\lambda}^{(\beta)}$ and on the change in the test medium refractive index of the $S_{n}^{(\beta)}$, as well as the width of the angular resonance characteristic $\delta \theta_{0.5}$. The angular dependences of the reflection coefficient on the angle of incidence $\theta$ for three different sensitive sensor elements are shown in Figure 4. The resonance curves were calculated numerically; in particular, for the prism structure, by the matrix method [43], and, for grating structures, by the Rigorous couple wave method (RCWA) [47]. The modulation amplitude of the refractive index for the dielectric grating is 0.017 . The values for $\delta \theta_{0.5}$ are presented in column 6 of Table 2 .

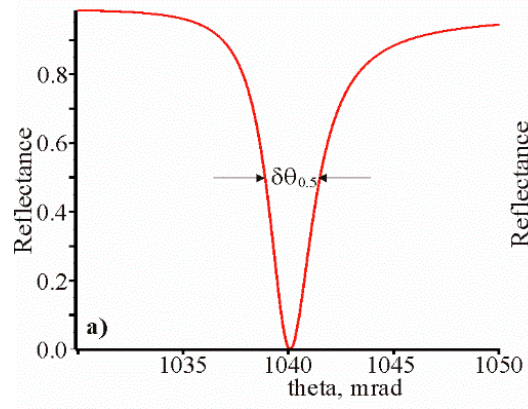

(a)

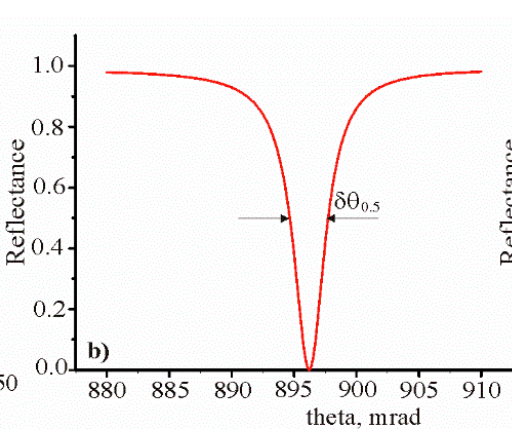

(b)

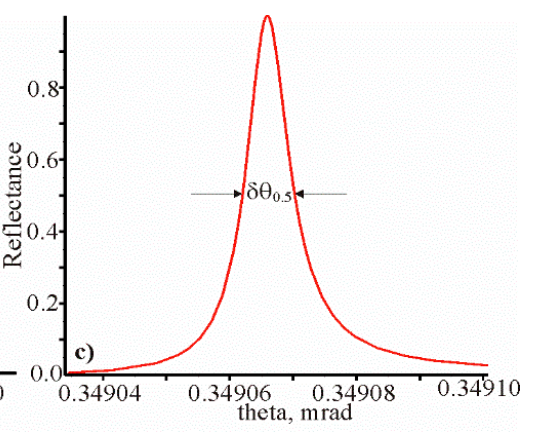

(c)

Figure 4. Angular dependences of the reflection coefficient of the light beam with the wavelength of $1.064 \mu \mathrm{m}$ for sensor elements presented in Figure 1a (a), in Figure 1b (b) and in Figure 1c (c).

The main parameters of the resonance structures are presented in columns 1,2 and 3 of Table 2 . The columns from 4 to 8 show the parameters obtained by the matrix method (row 3 ) and Rigorous coupled wave analysis (RCWA) (rows 4 and 5). It can be seen that the data of columns 9 and 10 in the corresponding rows are practically the same. The angular sensitivities (column 8 ) of the prism structure and the metal grating are close to each other. The spectral sensitivity of the prism structure is much higher than for the metal grating. However, the ratios $\frac{S_{\lambda}}{\delta \lambda_{0.5}}$ and $\frac{S_{\theta}}{\delta \theta_{0.5}}$ are about the same for both structures. The spectral and angular sensitivities of the dielectric grating are approximately an order of magnitude lower than for the metal grating. At the same time, the ratios $\frac{S_{\lambda}}{\delta \lambda_{0.5}}$ and $\frac{S_{\theta}}{\delta \theta_{0.5}}$ are 
approximately 30 times larger than the corresponding ratios for the prism structure and the metal grating. It is due to the very narrow resonant spectral and angular dependencies.

Table 2. The parameters of the sensors' sensitive elements (columns 1-2) and their characteristics calculated numerically (columns 3-10).

\begin{tabular}{ccccccccccc}
\hline Parameter & $n_{\boldsymbol{a}}$ & $\boldsymbol{\Lambda}, \mathbf{n m}$ & $\boldsymbol{d}$ & $\boldsymbol{\theta}, \mathbf{m r a d}$ & $\delta \lambda_{0.5}, \mathbf{n m}$ & $\delta \theta_{0.5}, \mathbf{m r a d}$ & $S_{\boldsymbol{\lambda}}, \mathbf{n m}$ & $S_{\theta}, \mathbf{m r a d}$ & $\frac{S_{\lambda}}{\delta \lambda_{0.5}}$ & $\frac{S_{\theta}}{\delta \theta_{0.5}}$ \\
\hline No & $\mathbf{1}$ & $\mathbf{2}$ & $\mathbf{3}$ & $\mathbf{4}$ & $\mathbf{5}$ & $\mathbf{6}$ & $\mathbf{7}$ & $\mathbf{8}$ & $\mathbf{9}$ & $\mathbf{1 0}$ \\
\hline Figure 1a & 1.3242 & - & 52.7 & 1040.2 & 47.14 & 2.611 & 24,043 & 1327.8 & 510 & 509 \\
\hline Figure 1b & 1.3242 & 500 & 10.0 & 896.2 & 0.95 & 3.07 & 500 & 1605 & 526 & 523 \\
\hline Figure 1c & 1.5 & 570.35 & 1870 & 349.1 & 0.0044 & 0.008243 & 75.8 & 141.9 & 17,227 & 17,215 \\
\hline
\end{tabular}

Table 3 shows the calculation results. Data in columns 6-11 are obtained on basis of the data in columns 1-5. The results of column 3 are determined from the angular dependences of the reflection coefficient, which are shown in Figure 4. The data of columns 4 and 5 are obtained using Equations (7) and (8), where the propagation constants $\beta$ are determined from solution of the corresponding wave equations. The data of columns from 6 to 11 are calculated using equations presented in Table 1, columns 1 to 5 .

Table 3. The parameters of the sensors' sensitive elements (columns 1-3) and their characteristics calculated from the obtained equations (columns 6-9).

\begin{tabular}{ccccccccccc}
\hline Parameter & $n_{\boldsymbol{a}}$ & $\theta_{,} \mathbf{m r a d}$ & $\delta \theta_{0.5}, \mathbf{m r a d}$ & $S_{\lambda}^{(\beta)}, \mu \mathbf{m}^{-2}$ & $S_{n}^{(\beta)}, \mu \mathbf{m}^{-\mathbf{1}}$ & $\delta \lambda_{0.5}, \mathbf{n m}$ & $S_{\boldsymbol{\lambda}}, \mathbf{n m}$ & $S_{\theta}, \mathbf{m r a d}$ & $\frac{S_{\lambda}}{\delta \lambda_{0.5}}$ & $\frac{S_{\theta}}{\delta \theta_{0.5}}$ \\
\hline No & $\mathbf{1}$ & $\mathbf{2}$ & $\mathbf{3}$ & $\mathbf{4}$ & $\mathbf{5}$ & $\mathbf{6}$ & $\mathbf{7}$ & $\mathbf{8}$ & $\mathbf{1 0}$ & $\mathbf{1 1}$ \\
\hline Figure 1a & 1.3242 & 1040.2 & 2.611 & -7.725 & 6.190 & 47.33 & 24,056 & 1326.4 & 508 & 508 \\
\hline Figure 1b & 1.3242 & 896.2 & 3.07 & -7.35 & 5.910 & 0.959 & 500 & 1601 & 521 & 522 \\
\hline Figure 1c & 1.5 & 349.1 & 0.008243 & -8.48 & 0.7859 & 0.00442 & 75.9 & 141.6 & 17,172 & 17,178 \\
\hline
\end{tabular}

A comparison of the data in the columns 6-11 of Table 3 with the data in the Table 2 shows their coincidence with high accuracy. It confirms the reasonableness of the obtained analytical equations.

\section{Discussion}

All three studied structures are united by the fact that they are, in fact, planar waveguides. In the first two structures, a surface plasmon-polariton wave is excited, and in the third one, the localized waveguide mode is excited. In the case of waveguide resonance, the Equation (1) with careful selected parameters of the prism structure is accurate, so the planarity of the metal film is not violated. For grating structures, shown in Figure 1b,c, the Equations (2) and (3) are approximate for disturbances in the homogeneity of the layers. These disturbances of the layer homogeneity are small, because the grating thickness (Figure $1 \mathrm{~b}$ ) is small $(10 \mathrm{~nm})$, and the modulation amplitude of the refractive index of the dielectric grating (Figure 1c) is 0.017. The difference between the right and left sides of Equations (2) and (3) is negligible due to this fact. Bulk phase dielectric gratings based on photopolymer composites can be obtained by the holographic method. They are characterized by high diffraction efficiency, uniformity of parameters over the area and practically no absorption, and the modulation amplitude of the refractive index in the grating is less than 0.02 [35].

Thus, the spectral and angular sensitivity of the sensors, and the sensitivity of the change in the propagation constant of the waveguide modes on the change in the wavelength and the change in the tested medium refractive index, were analytically connected using the conditions for the resonance appearance in waveguide structures, Equations (1), (2) and (3). Moreover, the analytical equations are determined by the sensitivity of the change in the propagation constant on the change in the tested medium refractive index for both grating structures (see Table 1). It is shown that the obtained analytical equations are practically the same for all three types of sensors under certain assumptions. In addition, analytical equations show that sensitivities increase when wavelength, grating period and 
sensitivity $S_{n}^{(\beta)}$ of change in the propagation constant on the change in the tested medium refractive index increase. It should be noted that sensitivities are inversely proportional to $\cos \theta$. It also was shown that, for all three structures, the equations connecting the characteristics of the sensors with the characteristics of the waveguides are the same.

The full widths at half maximum of the spectral and angular reflectance dependence, using the simple equation $\delta \lambda_{0.5}=n \Lambda \cos \theta_{0} \delta \theta_{00.5}$, were connected. Therefore, the following fundamental relation was obtained:

$$
\frac{S_{\lambda}}{\delta \lambda_{0.5}}=\frac{S_{\theta}}{\delta \theta_{00.5}}
$$

This equation is the most important for sensors, since it determines the suitability of the sensor for measuring changes in the tested medium refractive index. This ratio increases when wavelength and $S_{n}^{(\beta)}$ increase. It is inversely proportional to $\cos \theta$.

The obtained analytical equations are confirmed by numerous calculations. The angular and spectral sensitivity, as well as the width of the resonance curves, were determined on their base. The results of numerical calculations are presented in Tables 2 and 3. The calculations showed that the angular sensitivities for the sensors, which are shown in Figure $1 \mathrm{a}, \mathrm{b}$, are approximately the same. However, the spectral sensitivity of the prism structure is much higher than the corresponding sensitivity of the metal grating on the metal substrate. At the same time, the ratio $\frac{S_{\lambda}}{\delta \lambda_{0.5}}=\frac{S_{\theta}}{\delta \theta_{00.5}}$ is the same for both types of sensors. It is approximately 509 and 523. Sensors based on the dielectric grating on the dielectric substrate have the lowest angular and spectral sensitivity. There are $141.6 \mathrm{mrad}$ and $75.9 \mathrm{~nm}$, correspondingly. It should be noted that ratio $\frac{S_{\lambda}}{\delta \lambda_{0.5}}=\frac{S_{\theta}}{\delta \theta_{00.5}}$ is the largest among all types of the sensitive structures. This ratio is approximately of 17,200 for our research case. It is due to the very small widths of the resonance curves. To increase the width of the resonance curves, it is necessary to increase the modulation amplitude of the dielectric grating refractive index [4].

The measurement accuracy of the above-mentioned sensors is also determined by secondary devices, as well as by the methods of processing the received signals. Comparison of the sensors shown in Figure 1a,b, according to Table 3, shows that the sensor based on the prism structure has the highest spectral sensitivity. However, $\delta \lambda_{0.5}$ is higher for this sensor. At the same time, the ratio $\frac{S_{\lambda}}{\delta \lambda_{0.5}}$ is about the same for both sensors. The prism structure has an advantage over the grating structure shown in Figure $1 \mathrm{~b}$ if it is possible, with a secondary device and the corresponding software, to record the minimum of resonance with high accuracy. It should also be taken into account that the technology for manufacturing the prism structure is simpler than the technology for the grating structure. The characteristics of the prismatic structure can be improved, as suggested in [21,22].

The characteristics of the sensor shown in Figure $3 c$ differ significantly from the first two cases. The spectral and angular sensitivities are not high (see Table 3), however, $\frac{S_{\lambda}}{\delta \lambda_{0.5}}$ is the highest (see Table 3 ). That is, if a secondary device can record a minimum of resonance curves with a high resolution, then a sensor based on the dielectric grating can record the slightest changes in the refractive index of the tested medium.

In our work, we have described the simplest sensitive structures, however, there are possible ways to complicate and improve them. The sensitivity can be increased by increasing the intensity of the electromagnetic field at the sensitive structure/tested medium interface or by increasing the contact area between two media on this interface. Increases in the field for the structure shown in Figure $3 c$ can be achieved by introducing the additional buffer layer with the minimum possible refractive index and thickness of $0.5-1 \mu \mathrm{m}$ between the grating and the substrate. It is possible to improve of the characteristics of the structure shown in Figure $1 \mathrm{~b}$ by forming the additional grating with width $\mathrm{F} \Lambda$ and a period that is much smaller than the wavelength, similarly to the prism structure as shown in [22].

It should be mentioned that the effect of temperature is one of the important factors determining the accuracy of measurements. As it is known, it determines a lot of the dimensional errors of the measurement result [48]. Moreover, the temperature substantially affects the material parameters of the sensor elements. In our previous work [49], it was indicated that the reflectance spectra in sensors 
are shifted to the region of the smaller resonance angles of the incident wave with the increase in temperature. In addition, the resonance curves are broadened out when the temperature is increased. In general, we can say that temperature changes influence the resulting characteristics of optical sensors through the modulation of the refractive indices and the thickness of the materials used in the researched structure. The temperature's influence on the metal has a decisive role in the resonance of the reflectance curve. However, such impact of the temperature is significant only at high temperatures.

\section{Conclusions}

The optical characteristics of the planar sensors based on metal film, the metal grating on the metal substrate, and the dielectric grating on the dielectric substrate, has been researched. The relationship between the parameters of the sensor based on the prism structure, and the properties of the planar waveguide dielectric/thin metal film/dielectric, was obtained. Equations for the sensitivity of the sensor based on the metal grating under resonant excitation of the surface plasmon-polariton wave, and the sensor based on the dielectric grating under resonant excitation of the localized waveguide mode, were determined. The obtained analytical equations are confirmed by numerous calculations. The angular and spectral sensitivity, as well as the width of the resonance curves, were determined on their basis. Comparison of the properties of sensors based on planar waveguides showed that the main sensor characteristics are determined by the sensitivity of the constant propagation to a change in both the wavelength and the refractive index of the tested medium.

Author Contributions: S.B. planned the work and has been involved in writing and drafting the manuscript. O.V., V.F. and Y.B. have been involved in obtaining the new analytical relationships of angular and spectral sensitivities with the change of the refractive index of the medium. A.B. and I.Y. performed theoretical calculations of spectral characteristics and drafted, wrote, and arranged the article. All authors have read and agreed to the published version of the manuscript.

Funding: This work was supported, in the framework of the NATO Science for Peace and Security Programme, by the Multi-years Project "Nanocomposite Based Photonic Crystal Sensors of Biological and Chemical Agents"-grant SPS G5351-as well as partly by state budget funding in the framework of research work DB/MEV(No 0118U000267).

Conflicts of Interest: The authors declare no conflict of interest.

\section{References}

1. Xu, Y.; Bai, P.; Zhou, X.; Akimov, Y.; Png, C.E.; Ang, L.K.; Knoll, W.; Wu, L. Optical Refractive Index Sensors with Plasmonic and Photonic Structures: Promising and Inconvenient Truth. Adv. Opt. Mater. 2019, 7, 1801433. [CrossRef]

2. Quaranta, G.; Basset, G.O.; Martin, J.F.; Gallinet, B. Recent Advances in Resonant Waveguide Gratings. Laser Photonics Rev. 2018, 12, 1800017. [CrossRef]

3. Homola, J. Surface plasmon resonance sensors for detection of chemical and biological species. Chem. Rev. 2008, 108, 462-493. [CrossRef] [PubMed]

4. Wang, S.S.; Magnusson, R. Theory and applications of guided-mode resonance filters. Appl. Opt. 1993, 32, 2606-2613. [CrossRef] [PubMed]

5. Kretschmann, E.; Raether, H. Radiative decay of non radiative surface plasmons excited by light. Z. Naturforsch. A 1968, 23, 2135-2136. [CrossRef]

6. Otto, A. Excitation of nonradiative surface plasma waves in silver by the method of frustrated total reflection. Z. Phys. A 1968, 216, 398-410. [CrossRef]

7. Puiu, M.; Bala, C. SPR and SPR imaging: Recent trends in developing nanodevices for detection and real-time monitoring of biomolecular events. Sensors 2016, 16, 870. [CrossRef] [PubMed]

8. Yoon, K.H.; Shuler, M.L.; Kim, S.J. Design optimization of nano-grating surface plasmon resonance sensors. Opt. Express 2006, 14, 4842-4849. [CrossRef] [PubMed]

9. Fitio, V.M.; Bobitski, Y.V. Resonance effects in a dielectric grating; total absorption of electromagnetic waves by dielectric grating on T. metal system. J. Opt. A Pure Appl. Opt. 2004, 6, 943-951. [CrossRef] 
10. Klantsataya, E.; Jia, P.; Ebendorff-Heidepriem, H.; Monro, T.M.; François, A. Plasmonic Fiber Optic Refractometric Sensors: From Conventional Architectures to Recent Design Trends. Sensors 2017, 17, 12. [CrossRef]

11. Slavík, R.; Homola, J.; Čtyroký, J.; Brynda, E. Novel spectral fiber optic sensor based on surface plasmon resonance. Sens. Actuators B Chem. 2001, 74, 106-111. [CrossRef]

12. Schuster, T.; Herschel, R.; Neumann, N.; Schaffer, C.G. Miniaturized Long-Period Fiber Grating Assisted Surface Plasmon Resonance Sensor. J. Lightwave Technol. 2012, 30, 1003-1008. [CrossRef]

13. Santos, D.F.; Guerreiro, A.; Baptista, J.M. Surface plasmon resonance sensor based on D-type fiber with a gold wire. Optik 2017, 139, 244-249. [CrossRef]

14. Slavik, R.; Homola, J. Ultrahigh resolution long range surface plasmon-based sensor. Sens. Actuators B Chem. 2007, 123, 10-12. [CrossRef]

15. Destouches, N.; Pommier, J.C.; Parriaux, O.; Clausnitzer, T.; Lyndin, N.; Tonchev, S. Narrov band resonant grating of 100\% reflection under normal incedente. Opt. Express 2006, 14, 12613-12622. [CrossRef]

16. Anderson, B.B.; Brodsky, A.M.; Burgess, L.W. Threshold effects in light scattering from a binary diffraction grating. Phys. Rev. E 1996, 54, 912-923. [CrossRef]

17. Rosenblatt, D.; Sharon, A.; Friesem, A.A. Resonant Grating Waveguide Structures. IEEE J. Quantum Electron. 1997, 33, 2038-2059. [CrossRef]

18. Awazu, K.; Rockstuhl, C.; Fujimaki, M.; Fukuda, N.; Tominaga, J.; Komatsubara, T.; Ikeda, T.; Ohki, Y. High sensitivity sensor made of perforated waveguides. Opt. Express 2007, 15, 2592-2597. [CrossRef]

19. Fitio, V.; Yaremchuk, I.; Bobitski, Y. Optical excitation of surface plasmon polariton and waveguide modes resonances on prismatic structures. Opt. Appl. 2011, 4, 929-939.

20. Ran, B.; Lipson, S.G. Comparison between sensitive of phase and intensity detection in surface plasmon resonance. Opt. Express 2006, 14, 5641-5650. [CrossRef]

21. Ayushi, P.; Tomar, M.; Gupta, V. Refractive index sensor using long-range surface plasmon resonance with prism coupler. Plasmonics 2019, 14, 375-381.

22. Arora, P.; Talker, E.; Mazurski, N.; Levy, U. Dispersion engineering with plasmonic nano structures for enhanced surface plasmon resonance sensing. Sci. Rep. 2018, 8, 1-9.

23. Zeng, Y.; Hu, R.; Wang, L.; Gu, D.; He, J.; Wu, S.Y.; Ho, H.P.; Li, X.; Qu, J.; Gao, B.Z.; et al. Recent advances in surface plasmon resonance imaging: Detection speed, sensitivity, and portability. Nanophotonics 2017, 6, 1017-1030. [CrossRef]

24. Adam, P.; Dostálek, J.; Homola, J. Multiple surface plasmon spectroscopy for study of biomolecular systems. Sens. Actuators B Chem. 2006, 113, 774-781. [CrossRef]

25. Monteiro, J.P.; Ferreira, J.; Sabat, R.G.; Rochon, P.; Santos, M.J.L.; Girotto, E.M. SPR based biosensor using surface relief grating in transmission mode. Sens. Actuators B Chem. 2012, 174, 270-273. [CrossRef]

26. Vukusic, P.S.; Bryan-Brown, G.P.; Sambles, J.R. Surface plasmon resonance on gratings as a novel means for gas sensing. Sens. Actuators B Chem. 1992, 8, 155-160. [CrossRef]

27. Jory, M.J.; Vukusic, P.S.; Sambles, J.R. Development of a prototype gas sensor using surface plasmon resonance on gratings. Sens. Actuators B Chem. 1994, 17, 203-209. [CrossRef]

28. Choi, B.; Dou, X.; Fang, Y.; Phillips, B.M.; Jiang, P. Outstanding surface plasmon resonance performance enabled by templated oxide gratings. Phys. Chem.Chem. Phys. 2016, 18, 26078-26087. [CrossRef]

29. Dou, X.; Chung, P.Y.; Jiang, P.; Dai, J. Surface plasmon resonance and surface-enhanced Raman scattering sensing enabled by digital versatile discs. Appl. Phys. Lett. 2012, 100, 041116. [CrossRef]

30. Homola, J.; Koudela, I.; Yee, S.S. Surface plasmon resonance sensors based on diffraction gratings and prism couplers: Sensitivity comparison. Sens. Actuators B Chem. 1999, 54, 16-24. [CrossRef]

31. Piliarik, M.; Vala, M.; Tichý, I.; Homola, J. Compact and low-cost biosensor based on novel approach to spectroscopy of surface plasmons. Biosens. Bioelectron. 2008, 24, 3430-3435. [CrossRef] [PubMed]

32. Tamulevic `ius, T.; Seperys, R.; Andrulevicius, M.; Tamulevicius, S. Total internal reflection based sub-wavelength grating sensor for the determination of refractive index of liquids. Photonics Nanostruc.-Fundam. Appl. 2011, 9 , 140-148. [CrossRef]

33. Yaremchuk, I.; Tamulevičius, T.; Fitio, V.; Gražulevičiūte, I.; Bobitski, Y.; Tamulevičius, S. Guide-mode resonance characteristics of periodic structure on base of diamond-like carbon film. Opt. Commun. 2013, 6, 1-6. [CrossRef]

34. Snyder, A.W.; Love, J.D. Optical Waveguide Theory; Chapman and Hall: London, UK; New York, NY, USA, 1983. 
35. Fitio, V.M.; Sakhno, O.V.; Smirnova, T.N. Analysis of the diffraction by the gratings generated in the materials with a nonlinear response. Optik 2008, 119, 236-246. [CrossRef]

36. Fitio, V.M.; Laba, H.P.; Bobitski, Y.W. Absorption of Electromagnetic Waves into Periodic Structure and Thin Film of Metal when a Resonance of Plasmons Appears as a Result of Prism Excitation. Telecommun. Radio Eng. 2007, 66, 607-618. [CrossRef]

37. Johnson, P.B.; Christy, R.W. Optical Constants of the Noble Metals. Phys. Rev. B 1972, 6, 4370-4379. [CrossRef]

38. Treacy, M.M.J. Dynamical diffraction explanation of the anomalous transmission of light through metallic gratings. Phys. Rev. B 2002, 66, 195105. [CrossRef]

39. Kedenburg, S.; Vieweg, M.; Gissibl, T.; Giessen, H. Linear refractive index and absorption measurements of nonlinear optical liquids in the visible and near-infrared spectral region. Opt. Mat. Express 2012, 2, 1588-1611. [CrossRef]

40. Daimon, M.; Masumura, A. Measurement of the refractive index of distilled water from the near-infrared region to the ultraviolet region. Appl. Opt. 2007, 46, 3811-3820. [CrossRef]

41. Fitio, V.M.; Romakh, V.V.; Bobitski, Y.V. Numerical method for analysis of waveguide modes in planar gradient waveguides. Mater. Sci. (Medžiagotyra) 2014, 20, 256-261. [CrossRef]

42. Fitio, V.M.; Bendzyak, A.V.; Yaremchuk, I.Y.; Bobitski, Y.V. Wave equation solution for multilayer planar waveguides in a spatial frequency domain. Semicond. Phys. Quantum Electron. Optoelectron. 2017, 20, 424-429. [CrossRef]

43. Dobrowolski, J.A. Optical properties of films and coatings. In Handbook of Optics; McGREAW-HILL: New York, NY, USA, 1995; Volume 1, pp. 42-43.

44. Maier, S.A. Plasmonics: Fundamentals and Applications; Springer Science \& Business Media: Berlin, Germany, 2007.

45. Avrutsky, I.A.; Sychugov, V.A. Reflection of a Beam of Finite Size from a Corrugated Waveguide. J. Mod. Opt. 2007, 36, 1527-1539. [CrossRef]

46. Bellucci, S.; Vernyhor, O.; Bendziak, A.; Yaremchuk, I.; Fitio, V.M.; Bobitski, Y. Characteristics of the Surface Plasmon-Polariton Resonance in a Metal Grating, as a Sensitive Element of Refractive Index Change. Materials 2020, 13, 1882. [CrossRef] [PubMed]

47. Moharam, M.G.; Gaylord, T.K. Rigorous coupled-wave analysis of metallic surface-relief grating. J. Opt. Soc. Am. A 1986, 3, 1780-1787. [CrossRef]

48. Dorozinska, H.V.; Turu, T.A.; Markina, O.M.; Dorozinsky, G.V.; Maslov, V.P. Influence of Temperature on the Measuring Accuracy of Devices Based on Surface Plasmon Resonance Phenomenon. Mod. Instrum. 2018, 7, 1-10. [CrossRef]

49. Yaremchuk, I.; Fitio, V.; Petrovska, H.; Bobitski, Y. The temperature impact on the characteristics of the surface plasmon resonance sensors element. Optik 2019, 192, 162969. [CrossRef] 